\title{
An unusual case of pseudomyxoma peritonei associated with synchronous primary mucinous tumors of the ovary and appendix: A case report
}

\author{
DONG SOO SUH ${ }^{1,2^{*}}$, YONG JUNG SONG ${ }^{3 *}$, BYUNG SU KWON ${ }^{1,2}$, \\ SUL LEE ${ }^{1}$, NAM KYUNG LEE ${ }^{2,4}$, KYUNG UN CHOI ${ }^{5}$ and KI HYUNG KIM ${ }^{1,2}$
}

${ }^{1}$ Department of Obstetrics and Gynecology, Pusan National University School of Medicine; ${ }^{2}$ Biomedical Research Institute and Pusan Cancer Center, Pusan National University Hospital, Busan 49241; ${ }^{3}$ Research Institute for Convergence of Biomedical Science and Technology, Pusan National University Yangsan Hospital, Yangsan, South Gyeongsangnam 50612; Departments of ${ }^{4}$ Radiology and ${ }^{5}$ Pathology, Pusan National University School of Medicine, Busan 49241, Republic of Korea

Received September 29, 2016; Accepted February 28, 2017

DOI: $10.3892 / 01.2017 .6079$

\begin{abstract}
Pseudomyxoma peritonei (PMP) is a rare disease that usually originates from mucinous neoplasms of the appendix and, less commonly, from extra-appendiceal tumors, but it may also be caused by synchronous primary mucinous tumors of the ovary and appendix. The current study discusses the case of a 73-year-old female who presented with progressively worsening indigestion and abdominal distension. Magnetic resonance imaging of the pelvis revealed a large cystic mass with a thin enhancing septa arising from the right ovary and ascites with scalloping of the liver surface. On laparotomy, the patient was observed to have a large ruptured mass on the right ovary and a partially amputed distended appendix filled with mucin. The subsequent pathology report confirmed a borderline mucinous tumor of the right ovary and a low-grade appendiceal mucinous neoplasm associated with PMP. Immunohistochemical examination demonstrated the ovarian tumor stained strongly positive for cytokeratin (CK)-7, and negatively for CK-20 and homeobox protein CDX-2 (CDX2), whereas the appendiceal tumor stained negative for CK-7 and positive for CK-20 and CDX2. This study presents a rare case of PMP caused by synchronous primary mucinous tumors of the ovary and appendix in a postmenopausal female.
\end{abstract}

Correspondence to: Professor Ki Hyung Kim, Department of Obstetrics and Gynecology, Pusan National University School of Medicine, 179 Gudeok Street, Seo, Busan 49241, Republic of Korea E-mail: ghkim@pusan.ac.kr

*Contributed equally

Key words: pseudomyxoma peritonei, mucinous neoplasms, appendix, ovary

\section{Introduction}

Pseudomyxoma peritonei (PMP) is a rare disease caused by primary mucinous tumors that arise from different sites, usually from the appendix or ovary (1). It is estimated to occur in 1 to 2 individuals per million (2). PMP is a fatal clinical syndrome that may be associated with pelvic/ovarian masses and abundant mucinous ascites, caused by the rupture or leakage of a mucinous neoplasm within the abdomen (3). If untreated, patients frequently succumb to malnutrition due to the compression and blockage of the digestive tract by accumulating intraperitoneal mucin; however, significant invasive surgical procedures to treat PMP are associated with numerous complications and morbidities (3). Usually a diagnosis is produced with ultrasonography, computed tomography (CT), magnetic resonance imaging (MRI) and immunohistochemical markers.

The coexistence of mucinous appendiceal and ovarian tumors is commonly exhibited in PMP; however, the origin of these tumors in such patients is the subject of considerable debate; a mucinous ovarian tumor, in the presence of PMP, is likely to be of appendiceal origin (4). The identification of two truly independent primary mucinous tumors involving the appendix and ovary associated with PMP is uncommon. Sites of origin may be determined based on clinical, pathological and immunohistochemical features. The current study presents a rare case of PMP caused by synchronous primary mucinous tumors of the ovary and appendix in a 73 -year-old female.

\section{Case report}

A 73-year-old female was referred to the Department of Gynecology of the Pusan National University Hospital on 28 July 2015 for the evaluation of a pelvic mass, and presented with progressively worsening indigestion and abdominal distension. Ultrasound examination of the pelvis and abdomen revealed a large multiseptated cystic mass on the right ovary with a large amount of peritoneal fluid in 
the upper abdomen. Expression levels of the tumor markers cancer antigen (CA) 125 and human epididymis protein 4 were elevated to $51.79 \mathrm{U} / \mathrm{ml}$ and $98.07 \mathrm{ng} / \mathrm{ml}$, respectively, and the risk of ovarian malignancy algorithm was $\sim 39.38 \%$, suggestive of malignancy. MRI was performed to characterize the pelvic mass, and a T2-weighted MRI revealed a large multilocular cystic mass, measuring $21.8 \mathrm{~cm}$. No mural nodules or enhancing solid components were observed within the mass; however, ascites and mild scalloping of the liver surface were evident. Diffusion-weighted images (DWI) with a b-value of $400 \mathrm{~s} / \mathrm{mm}^{2}$ demonstrated hypointense septa in the fluid collection at the cul-de-sac (Fig. 1). The presumed preoperative diagnosis was PMP associated with an ovarian mucinous malignancy.

Laparotomy results demonstrated the presence of a large, well-circumscribed and ruptured cyst arising from the right ovary, a normal uterus and left ovary and considerable gelatinous mucinous peritoneal effusion occupying the whole pelvis and abdomen. The appendix was identified to be partially amputed following a previous rupture, distended and filled with mucin (Fig. 2). Bilateral salpingo-oophorectomy, hysterectomy, ileocecectomy, omentectomy, excisions of multifocal peritoneal mucinous implants and peritoneal lavage were performed on 4 August 2015.

Microscopically, the ovarian tumor was a multicystic lesion with mucinous epithelium and mucin pools in the wall. The lining of the mucinous epithelium exhibited nuclear enlargement, stratification and a complex architecture. The appendiceal mucinous tumor contained a large mucin pool and the lining epithelium exhibited low-grade dysplasia, but did not demonstrate infiltrative growth. The final pathological diagnosis was a borderline mucinous tumor of the right ovary and a low-grade appendiceal mucinous neoplasm associated with PMP (Fig. 3).

Surgical specimens were submitted for immunohistochemical examinations to distinguish the origin of the tumor. Immunohistochemistry was performed on serial $4-\mu \mathrm{m}$ thick paraffin sections. The paraffin sections were deparaffinized in xylene and rehydrated with a descending ethanol series. Bond Epitope Retrieval Solution 1 ( $\mathrm{pH}$ 6; Leica Microsystems $\mathrm{GmbH}$, Wetzlar, Germany) was used for antigen retrieval for $20 \mathrm{~min}$ at $100^{\circ} \mathrm{C}$. Monoclonal CK7 (dilution, 1:100; cat. no., NCL-L-CK7-OVTL), CK20 (dilution, 1:100; cat. no., NCL-L-CK20-561; both from Novocastra; Leica Microsystems $\mathrm{GmbH}$ ) and CDX2 antibodies (dilution 1:250; cat. no., 235R-15; Sigma Alrich; Merck KGaA) were applied on the slides. Immunohistochemical staining was performed with a Leica Bond-MAX ${ }^{\mathrm{TM}}$ autostainer and a Peroxidase/DAB Bond $^{\mathrm{TM}}$ Polymer Refine Detection system was used for visualization (both Leica Microsystems GmbH). Slides were incubated with a peroxide block for $5 \mathrm{~min}$, the primary antibody for $15 \mathrm{~min}$, a post-primary reagent for $8 \mathrm{~min}$ and the polymer for $8 \mathrm{~min}$, at room temperature. Cells were considered positive for CK7 and CK20 when cytoplasm was stained, and positive for CDX2 when nuclear staining was observed, using a light microscope (BX53, Olympus Corporation, Tokyo, Japan). The ovarian tissue sample stained strongly positive for cytokeratin (CK)-7 and negative for CK-20 and homeobox protein CDX-2 (CDX2), whereas the appendiceal tumor stained negative for CK-7 and positive for CK-20 and CDX2 (Fig. 4).
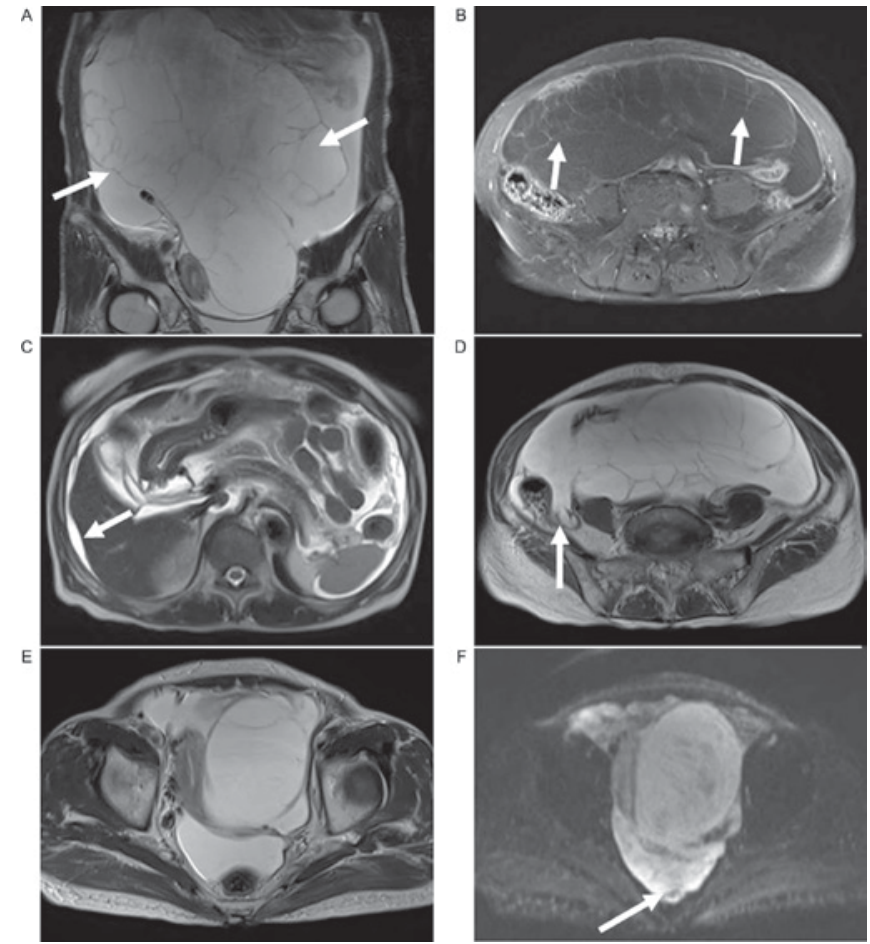

Figure 1. MRI features of pseudomyxoma peritonei associated with synchronous mucinous tumors of the ovary and appendix. (A) T2-weighted image exhibiting a $21-\mathrm{cm}$ multiseptated cystic mass (arrows) in the pelvic cavity. (B) Contrast enhanced, fat-suppressed T1-weighted image in which the cystic mass reveals an enhancement of the septa (arrows) without an enhancing solid portion in the cyst wall. (C) T2-weighted image of the upper abdomen demonstrated ascites with visceral scalloping (arrow) of the liver surface. (D) T2-weighted image revealing a 1-cm tubular cystic structure adjacent to the cecum, suggestive of appendiceal mucocele. However, the appendiceal mucocele was missed in preoperative MRI. (E) T2-weighted image of the pelvis indicating hyperintense fluid collection in the cul-de-sac. (F) Diffusion-weighted image of the pelvis with a b-value of $400 \mathrm{~s} / \mathrm{mm}^{2}$ revealing hypointense septa (arrow) in the fluid collection. MRI, magnetic resonance imaging.

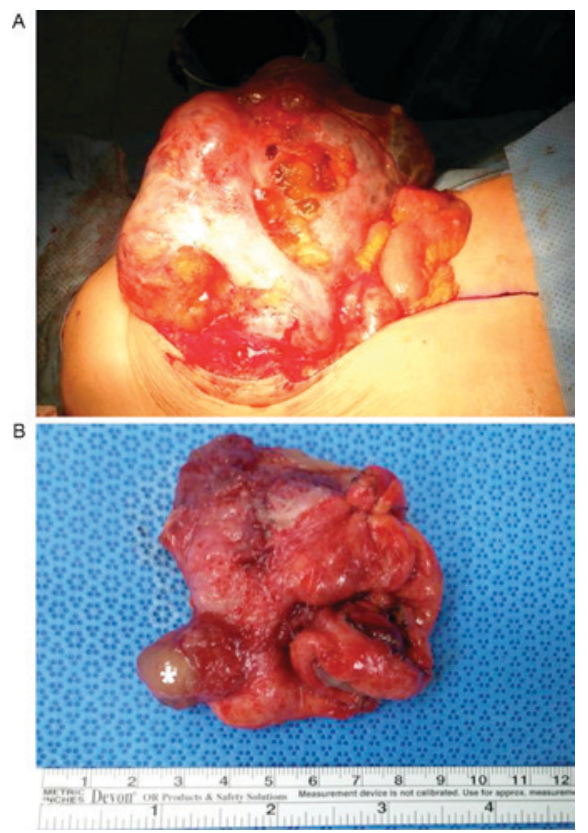

Figure 2. Intraoperative images of (A) the cystic tumor of the ovary and mucinous peritoneal effusion and of (B) the resected ileocecum with a small, partially amputed from a previous rupture, distended appendix filled with mucin (indicated with a white asterisk). 

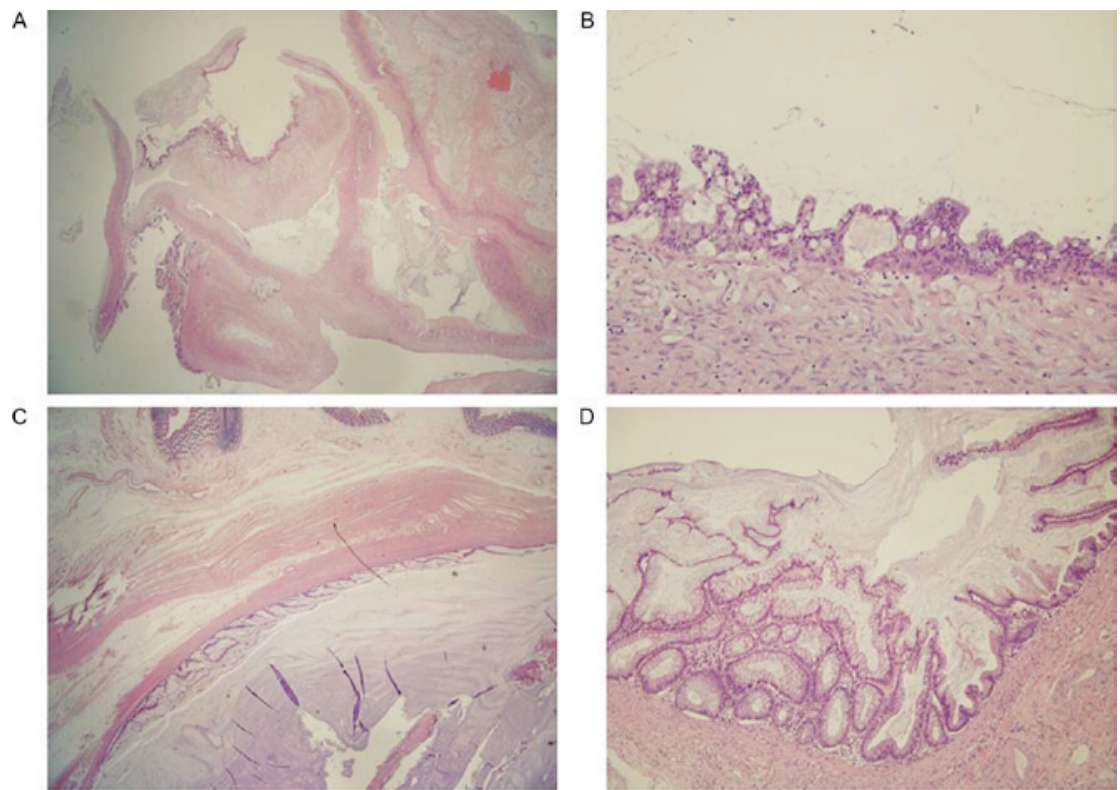

Figure 3. (A) Image demonstrating the multicystic nature of the ovarian tumor with mucinous epithelium and mucin pools in the wall. Magnification, x10. (B) Lining of the mucinous epithelium exhibited nuclear enlargement, stratification and a complex architecture. Magnification, x200. (C) The appendiceal mucinous tumor contained a large mucin pool (magnification, x10), and (D) its lining epithelium exhibited low-grade dysplasia but no infiltrative growth (magnification, x100). Hematoxylin and eosin staining was used for all images.

A

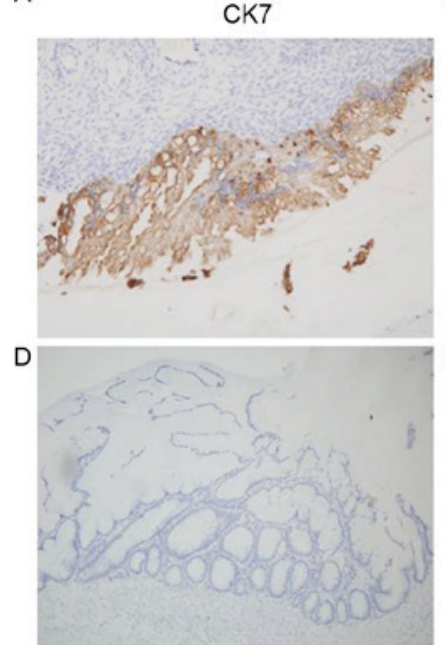

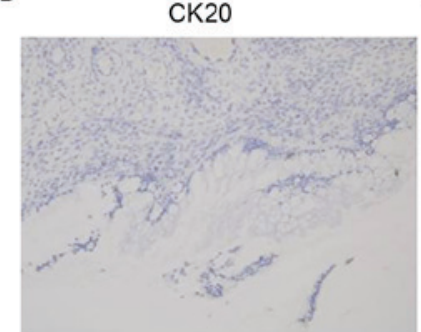

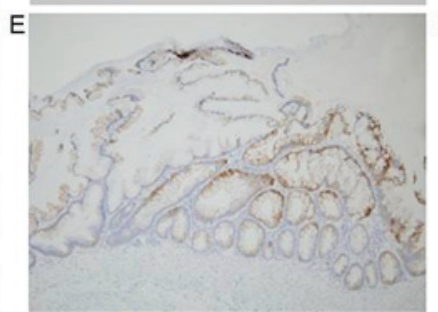

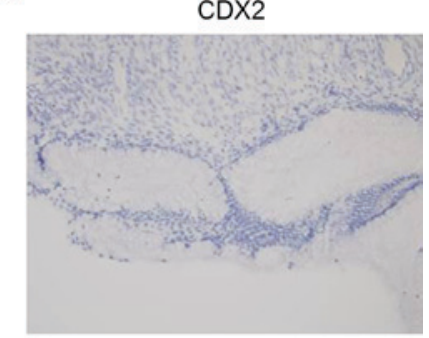

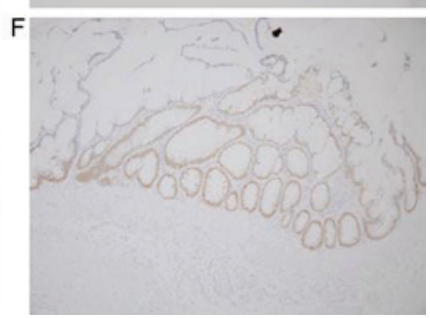

Figure 4. Immunohistochemical staining of the appendiceal and ovarian masses. The tumor cells of the right ovarian mass were (A) strongly positive for CK7, and negative for (B) CK20 and (C) CDX2. The tumor cells of the appendiceal mass were (D) negative for CK7 and positive for (E) CK20 and (F) CDX2. CK, cytokeratin; CDX2, homeobox protein CDX-2.

The patient's postoperative course was uneventful and she was discharged in a stable condition. Follow up was performed using CT imaging and serial measurement of serum carcinoembryonic antigen (CEA) and CA19-9 tumor markers. The CEA and CA19-9 levels were determined using radioimmunoassay kits manufactured from Abbott Laboratories (Chicago, IL, USA). The CEA and CA19-9 levels of the patient remained within normal levels and no recurrence was observed during the first 14 months subsequent to surgery.

\section{Discussion}

Pseudomyxoma peritonei is almost always associated with a mucinous neoplasm of the appendix (5), and is also commonly associated with an ovarian mucinous tumor. PMP exhibits well-established clinical characteristics, including a protracted clinical course, multiple recurrences and progressive fibrous adhesions leading to fatal intestinal obstruction. Previously, aggressive cytoreductive surgery (CRS) and hyperthermic intra-peritoneal chemotherapy (HIPEC) have been suggested to improve clinical outcomes and survival (6).

Considerable controversy surrounds the origin of this disease; the coexistence of mucinous tumors of the ovary and appendix associated with PMP is commonly encountered (4). Globally, $\sim 1 / 3$ to $1 / 2$ of women with PMP exhibit concurrent ovarian and appendiceal mucinous tumors (7). Guo et al reported that concurrent ovarian and appendiceal lesions occurred in 20/35 cases (7). However, the origin of 
the tumor in cases of synchronicity is widely debated (8). Clinicopathological and immunohistochemical data suggest that synchronous mucinous tumors of the ovary and appendix in women with PMP are derived from the appendix (9-11). Patients with ovarian and appendiceal tumors of independent origins have been identified (4). However, the presence of two truly independent primary mucinous tumors involving the appendix and ovary associated with PMP is uncommon.

It is now understood that primary mucinous tumors are much less common than previously considered, and that metastatic mucinous tumors from other organs are common (12). Several criteria help to distinguish primary ovarian mucinous tumors from metastatic mucinous tumors: A large size $(>10 \mathrm{~cm})$, unilaterality, the presence of benign or borderline areas, an expansile pattern of invasion, a smooth surface and the absence of extra-ovarian disease, a low grade and low stage at presentation and less aggressive behavior all indicate a primary ovarian neoplasm, whereas bilateral ovarian involvement, smaller size, ovarian surface involvement, multiple nodules and an infiltrative pattern of stromal invasion favor an extra-ovarian origin (11-14). In the case of the present study, the ovarian tumor was a large, unilateral and low-grade tumor, which is consistent with a primary mucinous tumor of the ovary.

Previous evidence based on pathological, molecular and immunohistochemical features suggest that an appendiceal origin is the primary etiology of PMP in the majority of cases (15). Confirmation of a pathologically normal appendix is essential for determining an extra-appendiceal origin, and certain immunohistochemical markers, CK7, CK20 and CDX-2, may assist in determining the origin of an ovarian tumor (16). CK7+/CK20-CDX2- and CK7-/CK20+CDX2+ patterns are typical of epithelial ovarian and intestinal tumors, respectively (6). For the patient in this case study, the presence of these immunohistochemical patterns indicated malignant processes of independent origins.

Imaging studies, principally CT and MRI, aid the diagnosis of PMP, which typically exhibits low or proteinaceous attenuation ascites, scalloping of the liver, and splenic margins and peritoneal implants that may cause extrinsic pressure on bowel loops. Additionally, amorphous and curvilinear calcifications are occasionally present in peritoneal implants (5). In particular, scalloping of the hepatic and splenic margins is diagnostic (5). In the case of the present study, a $21-\mathrm{cm}$ large multiseptated cystic mass was encountered in the pelvic/abdominal cavity with ascites and visceral scalloping of the liver surface. In all cases of PMP, the appendix must be closely observed for mucocele in cross-sectional images. The typical imaging results of appendiceal mucocele are a cystic, well-encapsulated mass, sometimes with mural calcification, in the expected location (17). However, in the present case study, appendiceal mucocele was missed when interpreting preoperative MR images. In a number of cases, it may be difficult to identify the originating appendiceal mucocele using imaging, as the residual appendix may be small or fibrosed subsequent to rupture (18). It must also be understood that the ovaries require careful evaluation in female patients with PMP.

In a recent study, it was suggested that DWI with low b-values may aid the diagnosis of PMP by providing a clear visualization of septa in the intra-abdominal fluid (19). In the present case, DWI with a b-value of $400 \mathrm{~s} / \mathrm{mm}^{2}$ facilitated the visualization of hypointense septa in the pelvic fluid, whereas T2-weighted images did not. This ability of DWI to visualize septa may be due to the reduced signal intensity of highly mobile water molecules at low b-values.

The diagnosis in the case of the present study was predicted subsequent to the preoperative MRI examination and was confirmed postoperatively, whereas the site of origin was determined immunohistochemically.

CRS combined with HIPEC has previously resulted in encouraging outcomes (6). The patient in the present study was treated with CRS, which consisted of bilateral salpingo-oophorectomy, hysterectomy, ileocecectomy, omentectomy, excision of multifocal peritoneal mucinous implants and peritoneal lavage. The site of origin does not affect the natural history of PMP, as appendiceal and extra-appendiceal neoplasms share prognoses and clinicopathological features (20).

In conclusion, based on its clinicopathological and immunohistochemical features, the case of the present study represents a PMP resulting from two separate malignant processes of primary mucinous tumors of the ovary and appendix. Therefore, radiologists and clinicians must be aware of the rare occurrence of synchronous mucinous tumors of the ovary and the appendix.

\section{Acknowledgements}

This research was supported by the Basic Science Research Program through the National Research Foundation of Korea (NRF) funded by the Ministry of Education (grant no., 2013R1A1A4A01 010141).

\section{References}

1. Chira RI, Nistor-Ciurba CC, Mociran A and Mircea PA: Appendicular mucinous adenocarcinoma associated with pseudomyxoma peritonei, a rare and difficult imaging diagnosis. Med Ultrason 18: 257-259, 2016.

2. Moran BJ and Cecil TD: The etiology, clinical presentation, and management of pseudomyxoma peritonei. Surg Oncol Clin N Am 12: 585-603, 2003.

3. Pillai K, Akhter J, Mekkawy A, Chua TC and Morris DL: Physical and chemical characteristics of mucin secreted by pseudomyxoma peritonei (PMP). Int J Med Sci 14: 18-28, 2017.

4. Chuaqui RF, Zhuang Z, Emmert-Buck MR, Bryant BR, Nogales F, Tavassoli FA and Merino MJ: Genetic analysis of synchronous mucinous tumors of the ovary and appendix. Hum Pathol 27: 165-171, 1996.

5. ZissinR,GayerG,Fishman A,Edelstein Eand Shapiro-Feinberg M: Synchronous mucinous tumors of the ovary and the appendix associated with pseudomyxoma peritonei: CT findings. Abdom Imaging 25: 311-316, 2000.

6. Baratti D, Kusamura S, Nonaka D, Cabras AD, Laterza B and Deraco M: Pseudomyxoma peritonei: Biological features are the dominant prognostic determinants after complete cytoreduction and hyperthermic intraperitoneal chemotherapy. Ann Surg 249: 243-249, 2009.

7. Guo AT, Song X, Wei LX and Zhao P: Histological origin of pseudomyxoma peritonei in Chinese women: Clinicopathology and immunohistochemistry. World J Gastroenterol 17: 3531-3537, 2011.

8. Guerrieri C, Frånlund B, Fristedt S, Gillooley JF and Boeryd B: Mucinous tumors of the vermiform appendix and ovary, and pseudomyxoma peritonei: Histogenetic implications of cytokeratin 7 expression. Hum Pathol 28: 1039-1045, 1997.

9. Szych C, Staebler A, Connolly DC, Wu R, Cho KR and Ronnett BM: Molecular genetic evidence supporting the clonality and appendiceal origin of Pseudomyxoma peritonei in women. Am J Pathol 154: 1849-1855, 1999. 
10. Young RH, Gilks CB and Scully RE: Mucinous tumors of the appendix associated with mucinous tumors of the ovary and pseudomyxoma peritonei. A clinicopathological analysis of 22 cases supporting an origin in the appendix. Am J Surg Pathol 15: 415-429, 1991.

11. Hart WR: Mucinous tumors of the ovary: A review. Int J Gynecol Pathol 24: 4-25, 2005.

12. Rouzbahman M and Chetty R: Mucinous tumours of appendix and ovary: An overview and evaluation of current practice. J Clin Pathol 67: 193-197, 2014.

13. Yemelyanova AV, Vang R, Judson K, Wu LS and Ronnett BM: Distinction of primary and metastatic mucinous tumors involving the ovary: Analysis of size and laterality data by primary site with reevaluation of an algorithm for tumor classification. Am J Surg Pathol 32: 128-138, 2008.

14. Zaino RJ, Brady MF, Lele SM, Michael H, Greer B and Bookman MA: Advanced stage mucinous adenocarcinoma of the ovary is both rare and highly lethal: A Gynecologic Oncology Group study. Cancer 117: 554-562, 2011.

15. Nonaka D, Kusamura S, Baratti D, Casali P, Younan R and Deraco M: CDX-2 expression in pseudomyxoma peritonei: A clinicopathological study of 42 cases. Histopathology 49: 381-387, 2006
16. Zhou F, Chen X, Li Y and Huang L: Two independent primary mucinous tumors involving the appendix and ovary accompanied with acellular pseudomyxoma peritonei. Int J Clin Exp Pathol 8: 11831-11834, 2015.

17. Levy AD, Shaw JC and Sobin LH: Secondary tumors and tumorlike lesions of the peritoneal cavity: Imaging features with pathologic correlation. Radiographics 29: 347-373, 2009.

18. Sugarbaker PH, Ronnett BM, Archer A, Averbach AM, Bland R, Chang D, Dalton RR, Ettinghausen SE, Jacquet P, Jelinek J, et al: Pseudomyxoma peritonei syndrome. Adv Surg 30: 233-280, 1996.

19. Himoto Y, Kido A, Fujimoto K, Kawada K, Kitai T, Sakai Y and Togashi K: A case of pseudomyxoma peritonei: Visualization of septa using diffusion-weighted images with low b values. Abdom Radiol (NY) 41: 1713-1717, 2016.

20. Baratti D, Kusamura S, Milione M, Pietrantonio F, Caporale M, Guaglio $M$ and Deraco M: Pseudomyxoma peritonei of extra-appendiceal origin: A comparative study. Ann Surg Oncol 23: 4222-4230, 2016. 